\title{
Recovering the spectrum of a low level signal from two noisy measurements using the cross power spectral density
}

\author{
Andrew J. Fleming, ${ }^{\text {a) }}$ Brett Ninness, ${ }^{\text {b) }}$ and Adrian Wills ${ }^{c}$ \\ School of Electrical Engineering and Computer Science, University of Newcastle, \\ Callaghan, NSW 2308, Australia
}

(Received 1 April 2013; accepted 3 July 2013; published online 8 August 2013)

\begin{abstract}
The article describes a method for estimating the spectrum or RMS value of a low-level signal corrupted by noise. If two identical sensors can be employed simultaneously and the additive noise sources are uncorrelated, the cross power spectrum can recover the power spectrum of the underlying signal. When using the Welch method to estimate the cross power spectrum, the estimation process is shown to be biased but consistent, with a variance that is inversely proportional to the number of data sets. The proposed technique is demonstrated experimentally to recover the vibration spectrum of a piezoelectric cantilever. The dual sensor method reduces the effective noise floor by three orders of magnitude and recovers spectral features that were otherwise lost in noise. (C) 2013 AIP Publishing LLC. [http://dx.doi.org/10.1063/1.4815982]
\end{abstract}

\section{INTRODUCTION}

The power spectrum is used ubiquitously throughout science and engineering to characterize the frequency content of time series data. ${ }^{1,2}$ Application examples include biomedical EEG processing, ${ }^{3}$ radar processing, ${ }^{4}$ geophysical monitoring, ${ }^{5}$ astronomy, ${ }^{6}$ ocean wave characterization, ${ }^{7}$ prediction of control system resolution, ${ }^{8,9}$ and calibration of single molecule manipulators. ${ }^{10}$ In such applications, the power spectrum must be estimated from a finite length of potentially noisy data. Due to the breadth of applications, a diverse range of estimation techniques have also evolved. ${ }^{11,12}$ These methods can be grouped into either parametric and non-parametric estimation categories. ${ }^{13}$ Parametric methods fit a model to the measured data and are most useful when the model structure is at least partially known. Nonparametric methods do not assume a fixed model structure and estimate the power spectrum directly from measured data. Although this work focusses on nonparametric estimation, the concept is also applicable to parametric methods.

When estimating the spectrum of small signals, the measurement noise can be a prohibitive limitation. Consider the signal $\left\{d_{t}\right\}$ illustrated in Figure 1(a) which is corrupted by the additive noise process $\left\{e_{t}\right\}$. The power spectrum of the measurement $\left\{y_{t}\right\}$ is

$$
\Phi_{y}(\omega)=\Phi_{d}(\omega)+\Phi_{e}(\omega)
$$

where $\Phi_{y}(\omega)$ is the power spectrum of $\left\{y_{t}\right\}$. Equation (1) can be rearranged to reveal $\Phi_{d}$, however, this requires exact knowledge of the noise spectrum $\Phi_{e}(\omega)$ which is impractical. If the noise spectrum is unknown, the spectrum of $\left\{y_{t}\right\}$ cannot be recovered, especially if the noise is of comparable amplitude to the signal.

This article describes a dual sensor method for recovering the power spectrum of a signal lost in measurement

\footnotetext{
a) andrew.fleming@newcastle.edu.au

b)brett.ninness@newcastle.edu.au

c) adrian.g.wills@gmail.com
}

noise. This method is applicable when the underlying signal $\left\{d_{t}\right\}$ can be measured simultaneously using two sensors with uncorrelated noise processes as illustrated in Figure 1(b). In Sec. III, the spectrum of $\left\{d_{t}\right\}$ is shown to be equal to the cross power spectrum of two noisy measurements. Therefore, the spectrum can be fully recovered if the noise processes are uncorrelated.

When a finite number of samples are available, the Welch method is defined in Sec. V for estimating the cross power spectrum. The mean and variance of the dual sensor method is examined in Sec. VI. The dual sensor method is shown be a consistent estimator with a bias and variance that are both inversely proportional to the length of data available. Therefore, the spectrum of the underlying signal can be recovered to arbitrary accuracy given sufficient data length.

Not all applications allow for a second independent sensor, however, multiple sensors are routinely used in many precision measurement applications. ${ }^{14-17}$ Although these applications do not typically use two identical sensors, there is scope to do so.

The dual sensor method for estimating power spectrum is straightforward to implement in practice and provides a new tool for recovering the spectrum of small signals otherwise lost in measurement noise. It complements other signal recovery methods such as the lock-in amplifier that require a synchronous modulation of the measured variable. ${ }^{18,19}$

\section{QUASI-STATIONARY SIGNALS}

In general, the signals $\left\{d_{t}\right\},\left\{e_{t}\right\},\left\{v_{t}\right\},\left\{y_{t}\right\}$, and $\left\{u_{t}\right\}$ in Figure 1 may contain both deterministic and stochastic components. To address this situation, note that a signal $\left\{x_{t}\right\}$ is quasi-stationary $^{20}$ if it satisfies

$$
\begin{gathered}
\overline{\mathbf{E}}\left\{x_{t}\right\}=\bar{x}, \\
\overline{\mathbf{E}}\left\{\left(x_{t}-\bar{x}\right)\left(x_{t-\tau}-\bar{x}\right)\right\}=R_{x}(\tau),
\end{gathered}
$$


(a) Single Sensor
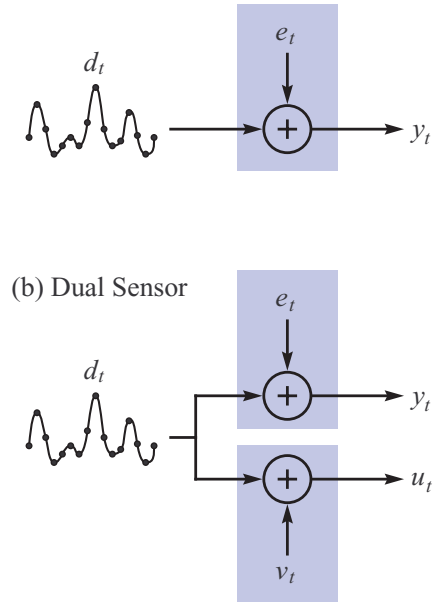

FIG. 1. The additive sensor noise of a single sensor (a) and dual sensor (b) The sensor contribution is shaded.

where $\overline{\mathbf{E}}\{\cdot\}$ is the time averaged expectation

$$
\overline{\mathbf{E}}\left\{x_{t}\right\} \triangleq \lim _{N \rightarrow \infty} \frac{1}{N} \sum_{t=1}^{N} \mathbf{E}\left\{x_{t}\right\}
$$

and $\mathbf{E}\{\cdot\}$ is the usual stochastic expectation operator. This definition allows stochastic and deterministic signals to be treated in the same manner. The associated power spectral density is defined as

$$
\Phi_{x}(\omega)=\sum_{\tau=-\infty}^{\infty} R_{x}(\tau) e^{-j \omega \tau} .
$$

Two signals $\left\{y_{t}\right\}$ and $\left\{u_{t}\right\}$ are known as jointly quasistationary if they are both stationary and the following crosscovariance function also exists ${ }^{20}$

$$
\overline{\mathbf{E}}\left\{\left(y_{t}-\bar{y}\right)\left(u_{t-\tau}-\bar{u}\right)\right\}=R_{y u}(\tau) .
$$

The cross power spectral density of two jointly quasistationary signals is defined as ${ }^{20}$

$$
\Phi_{y u}(\omega)=\sum_{\tau=-\infty}^{\infty} R_{y u}(\tau) e^{-j \omega \tau} .
$$

If $\left\{x_{t}\right\}$ is a wide sense stationary stochastic process, then via the above definitions

$$
\begin{gathered}
\bar{x}=\overline{\mathbf{E}}\left\{x_{t}\right\}=\mathbf{E}\left\{x_{t}\right\}, \\
\overline{\mathbf{E}}\left\{\left(x_{t}-\bar{x}\right)\left(x_{t-\tau}-\bar{x}\right)\right\}=\mathbf{E}\left\{\left(x_{t}-\bar{x}\right)\left(x_{t-\tau}-\bar{x}\right)\right\} .
\end{gathered}
$$

And hence the quasi-stationary mean, covariance, and power spectral density revert to the usual ones. However, consider the purely deterministic process

$$
x_{t}=\cos \omega_{0} t .
$$

Then clearly

$$
\overline{\mathbf{E}}\left\{x_{t}\right\}=0,
$$

and

$$
\begin{aligned}
\overline{\mathbf{E}}\left\{x_{t} x_{t-\tau}\right\} & =\lim _{N \rightarrow \infty} \frac{1}{N} \sum_{t=1}^{N} \cos \omega_{0} t \cos \omega_{0}(t-\tau) \\
& =\frac{1}{2} \lim _{N \rightarrow \infty} \frac{1}{N} \sum_{t=1}^{N} \cos \omega_{0} \tau+\cos \left(2 \omega_{0} t-\omega_{0} \tau\right) \\
& =\frac{1}{2} \cos \omega_{0} \tau
\end{aligned}
$$

and hence

$$
\Phi_{x}(\omega)=\lim _{N \rightarrow \infty} \sum_{\tau=-N}^{N} \frac{1}{2} \cos \omega_{0} \tau e^{-j \omega \tau}=\frac{\pi}{2} \delta\left(\omega \pm \omega_{0}\right) .
$$

\section{DUAL SENSOR METHOD}

If the signal $\left\{d_{t}\right\}$ can be measured simultaneously by two identical sensors, the signal path is illustrated in Figure 1(b). In this configuration, both sensors measure the same displacement but have uncorrelated additive noise sources $\left\{e_{t}\right\}$ and $\left\{v_{t}\right\}$, that is,

$$
\begin{aligned}
& y_{t}=d_{t}+e_{t}, \\
& u_{t}=d_{t}+v_{t} .
\end{aligned}
$$

If $\left\{d_{t}\right\}$ is quasi-stationary and $\left\{e_{t}\right\}$ and $\left\{v_{t}\right\}$ are zero mean stationary processes, the cross-correlation of $\left\{y_{t}\right\}$ and $\left\{u_{t}\right\}$ is

$$
R_{y u}(\tau)=\overline{\mathbf{E}}\left\{y_{t} u_{t+\tau}\right\},
$$

which is equal to

$$
\begin{aligned}
R_{y u}(\tau) & =\overline{\mathbf{E}}\left\{\left(d_{t}+e_{t}\right)\left(d_{t+\tau}+v_{t+\tau}\right)\right\} \\
& =\overline{\mathbf{E}}\left\{d_{t} d_{t+\tau}+d_{t} v_{t+\tau}+e_{t} d_{t+\tau}+e_{t} v_{t+\tau}\right\} .
\end{aligned}
$$

If the signals are uncorrelated, that is, if the cross-covariance is zero, $R_{y u}(\tau)$ reduces to the covariance of $\left\{d_{t}\right\}$, that is

$$
R_{y u}(\tau)=\overline{\mathbf{E}}\left\{d_{t} d_{t+\tau}\right\}=R_{d}(\tau) .
$$

By taking the Fourier transform of (21), the cross power spectral density of $\left\{y_{t}\right\}$ and $\left\{u_{t}\right\}$ is equal to the power spectral density of the underlying signal $\left\{d_{t}\right\}$, that is,

$$
\Phi_{y u}(\omega)=\Phi_{d}(\omega) .
$$

Thus, by using two independent sensors, the power spectral density of an underlying signal can be recovered from the cross-correlation or cross power spectral density of two noisy measurements. This is a convenient result as the cross power spectral density or cross-correlation can be readily measured in practice by a standard spectrum analyzer or from time domain recordings.

\section{ROOT-MEAN-SQUARE VALUE}

In some situations, only the Root-Mean-Square (RMS) value (or standard deviation) of a low-level signal is of interest. If the previous assumptions hold, the RMS value 
of $\left\{d_{t}\right\}$ is

$$
\sigma_{d}=\sqrt{R_{y u}(0)}, \quad \text { and } \quad \sigma_{d}=\sqrt{\overline{\mathbf{E}}\left\{y_{t} u_{t}\right\}} .
$$

Therefore, the RMS value of $\left\{d_{t}\right\}$ can be estimating from the time average of $\left\{y_{t}\right\} \times\left\{u_{t}\right\}$.

\section{WELCH ESTIMATE OF CROSS SPECTRAL DENSITY}

Practical application of Eq. (22) requires estimation of the cross power spectral density from a finite number of discrete time samples. The Welch estimate of spectral density involves averaging the spectrum of multiple windowed data records. ${ }^{21,22}$ If a data record of $y_{t}$ is split into $M$ sets of length $N$, the data are denoted $y_{k}^{i}$ where $k=1,2, \ldots, N$ and $i=1,2$, $\ldots, M$. Using this notation, the windowed DFT of the $i$ th data set of $\left\{y_{t}\right\}$ and $\left\{u_{t}\right\}$ is

$$
\begin{aligned}
& Y_{i}(\omega)=\frac{1}{N} \sum_{k=0}^{N-1} y_{k}^{i} w_{k} e^{-j \omega k}, \\
& U_{i}(\omega)=\frac{1}{N} \sum_{k=0}^{N-1} u_{k}^{i} w_{k} e^{-j \omega k}, \\
& D_{i}(\omega) \triangleq \frac{1}{N} \sum_{k=0}^{N-1} d_{k}^{i} w_{k} e^{-j \omega k},
\end{aligned}
$$

and we define

$$
W \triangleq \frac{1}{N} \sum_{k=0}^{N-1} w_{k}^{2}
$$

We then define the Welch estimate of the cross spectral density $\Phi_{y u}(\omega)$ according to

$$
\widehat{\Phi}_{y u}(\omega)=\frac{1}{M} \sum_{i=1}^{M} I_{i}, \quad I_{i}(\omega) \triangleq \frac{N}{W} Y_{i}(\omega) U_{i}^{\star}(\omega) .
$$

\section{PROPERTIES OF THE WELCH CROSS SPECTRUM ESTIMATE}

\section{A. Mean of the estimate}

First,

$$
\begin{aligned}
& \overline{\mathbf{E}}\left\{I_{i}(\omega)\right\} \\
& =\frac{1}{W} \sum_{k=0}^{N-1} \sum_{t=0}^{N-1} w_{k} w_{t} \overline{\mathbf{E}}\left\{y_{k}^{i} u_{t}^{i}\right\} e^{j \omega(t-k)} \\
& =\frac{1}{W} \sum_{k=0}^{N-1} \sum_{t=0}^{N-1} w_{k} w_{t}\left(\frac{1}{2 \pi} \int_{-\pi}^{\pi} \Phi_{y u}(\lambda) e^{j(k-t) \lambda} \mathrm{d} \lambda\right) e^{j \omega(t-k)} \\
& =\frac{1}{2 W \pi} \int_{-\pi}^{\pi} \Phi_{y u}(\lambda)\left|\sum_{k=0}^{N-1} w_{k} e^{-j k(\omega-\lambda)}\right|^{2} \mathrm{~d} \lambda
\end{aligned}
$$

Therefore,

$$
\overline{\mathbf{E}}\left\{\widehat{\Phi}_{y u}(\omega)\right\}=\frac{1}{M} \sum_{i=1}^{M} \overline{\mathbf{E}}\left\{I_{i}\right\}=\frac{1}{2 \pi} \int_{-\pi}^{\pi} \Phi_{y u}(\lambda) h(\omega-\lambda) \mathrm{d} \lambda,
$$

where

$$
h(\lambda) \triangleq \frac{1}{M W}\left|\sum_{k=0}^{N-1} w_{k} e^{-j k \lambda}\right|^{2}
$$

Therefore, the Welch estimate is a biased one, with the bias decreasing as the data length $N$ grows. The bias is due to a convolution of the true power spectrum and frequency response of the window function, which is identical to the standard Welch estimate. ${ }^{21}$

\section{B. Variance of the estimate}

Since both $\left\{y_{k}\right\}$ and $\left\{u_{k}\right\}$ are quasi-stationary processes, then for any fixed $\omega,\left\{I_{i}(\omega)\right\}$ must also be a quasi-stationary process $^{20}$ with respect to the index $i$. The associated covariance function is defined according to

$$
c_{\tau}=\overline{\operatorname{Cov}}\left\{I_{i}, I_{i+\tau}\right\} .
$$

In this case, with $\bar{I}_{i} \triangleq \overline{\mathbf{E}}\left\{I_{i}\right\}$, and .* indicating the complex conjugate,

$$
\begin{aligned}
\operatorname{Var}\left\{\widehat{\Phi}_{y u}\right\} & =\overline{\mathbf{E}}\left\{\left|\frac{1}{M} \sum_{i=1}^{M} I_{i}-\bar{I}_{i}\right|^{2}\right\} \\
& =\frac{1}{M^{2}} \sum_{i=1}^{M} \sum_{\ell=1}^{M} \overline{\mathbf{E}}\left\{\left(I_{i}-\bar{I}_{i}\right)\left(I_{\ell}-\bar{I}_{\ell}\right)^{\star}\right\} \\
& =\frac{1}{M^{2}} \sum_{i=1}^{M} \sum_{\ell=1}^{M} c_{i-\ell} \\
& =\frac{1}{M} \sum_{\tau=1-M}^{M-1}\left(1-\frac{|\tau|}{M}\right) c_{\tau} .
\end{aligned}
$$

The sum is the well-known Cesàro mean of the sequence $\left\{c_{\tau}\right\}$, for which a standard result ${ }^{23}$ is that

$$
\lim _{M \rightarrow \infty} \sum_{\tau=1-M}^{M-1}\left(1-\frac{|\tau|}{M}\right) c_{\tau}=\sum_{\tau=-\infty}^{\infty} c_{\tau} \triangleq \kappa
$$

provided this latter limit $\kappa$ exists. Assuming it does, then we have just established

$$
\lim _{M \rightarrow \infty} M \cdot \operatorname{Var}\left\{\widehat{\Phi}_{y u}\right\}=\kappa
$$

and hence we can expect the absolute error to satisfy

$$
\left|\widehat{\Phi}_{y u}-\overline{\mathbf{E}}\left\{\widehat{\Phi}_{y u}\right\}\right|=O\left(\frac{1}{\sqrt{M}}\right) .
$$

That is, the absolute error is inversely proportional to the square root of the number of data sets $M$. 


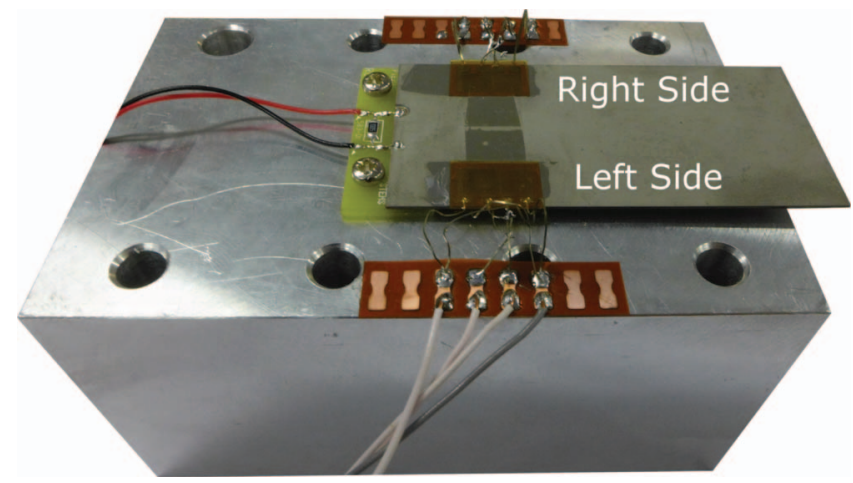

FIG. 2. The piezoelectric cantilever with base mounted strain gauges.

\section{EXPERIMENTAL DEMONSTRATION}

In this section, the cross power spectrum is employed to recover the vibration spectra of the piezoelectric cantilever pictured in Figure 2. The cantilever is a two-layer brassreinforced bimorph bender from Piezosystems Inc. (Q220A4-503YB). An applied voltage of $\pm 90 \mathrm{~V}$ results in a deflection of $\pm 1.25 \mathrm{~mm}$ at the tip.

Since the tip displacement of a cantilever beam is proportional to the base strain, the deflection can be estimated by mounting a strain gauge bridge at the base. Two identical strain gauge bridges are mounted on the left and right side of the bender. The left-side strain gauge circuit is illustrated in Figures 3(b) and 3(c). The bridge is completed with a $90^{\circ}$ rosette (Omega SGT-4/350-XY11) on the top surface (gauges 1 and 2) and another on the bottom surface (gauges 3 and 4). A downward tip displacement increases the resistance of gauges 1 and 3, and slightly reduces the resistance of gauges 3 and 4 . A practical signal amplitude was achieved by amplifying the differential strain signal by 5000 . The resulting sensitivity was $0.44 \mathrm{~mm} / \mathrm{V}$. Although the two strain gauge bridges generate a significant magnitude of thermal noise, ${ }^{24}$ the noise processes are uncorrelated.

To create a known vibration spectrum, the piezoelectric bender is excited with a $\pm 10 \mathrm{~V}$ triangular signal with a frequency of $11 \mathrm{~Hz}$. This excitation creates a decaying vibration spectrum consisting of odd-harmonics, that is, the frequency components will be $11 \mathrm{~Hz}, 33 \mathrm{~Hz}, 55 \mathrm{~Hz}, 77 \mathrm{~Hz}$, etc. The direct power spectrum estimate obtained from a spectrum analyzer is plotted in Figure 4. The noise floor of the sensor is approximately $3.8 \times 10^{-6} \mathrm{~V}^{2} / \mathrm{Hz}$, which is equivalent to $8.5 \mu \mathrm{m} / \sqrt{\mathrm{Hz}}$. Only the first five harmonics are clearly distinguishable; furthermore, the peak magnitudes are overestimated due to the high additive noise density.

With an identical number of averages $(M=100)$, the cross power spectral density reduces the noise floor by two orders of magnitude. This permits all of the harmonics to be clearly distinguished in addition to peaks resulting from ambient building vibration at $50 \mathrm{~Hz}$ and $150 \mathrm{~Hz}$. A minor peak at $90 \mathrm{~Hz}$ is also visible which is due to the acoustic noise generated by a nearby power supply fan.

When the input signal is reduced to $\pm 1 \mathrm{~V}$ the direct power spectrum, plotted in Figure 5(a), becomes dominated by the sensor noise. Only the first and fourth harmonics are (a) Bimorph excitation

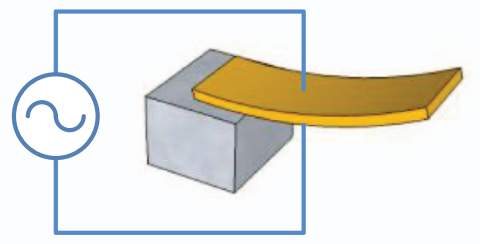

(b) Top view of left bridge

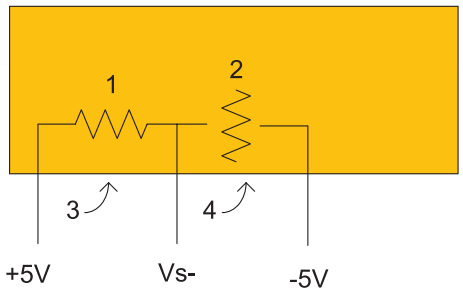

(c) Left bridge circuit

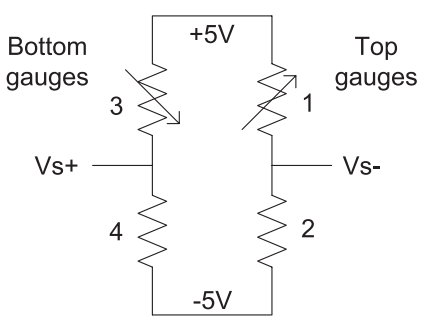

FIG. 3. The piezoelectric bender (a) and the left-side strain gauge bridge (b and $\mathrm{c}$ ).

clearly distinguishable. However, the lower noise floor of the dual sensor method allows the first six harmonics to be clearly identified but the higher harmonics remain lost in noise. By increasing the number of averages from to 1000 in Figures 5(b) and 10000 in 5(c), the noise floor is reduced

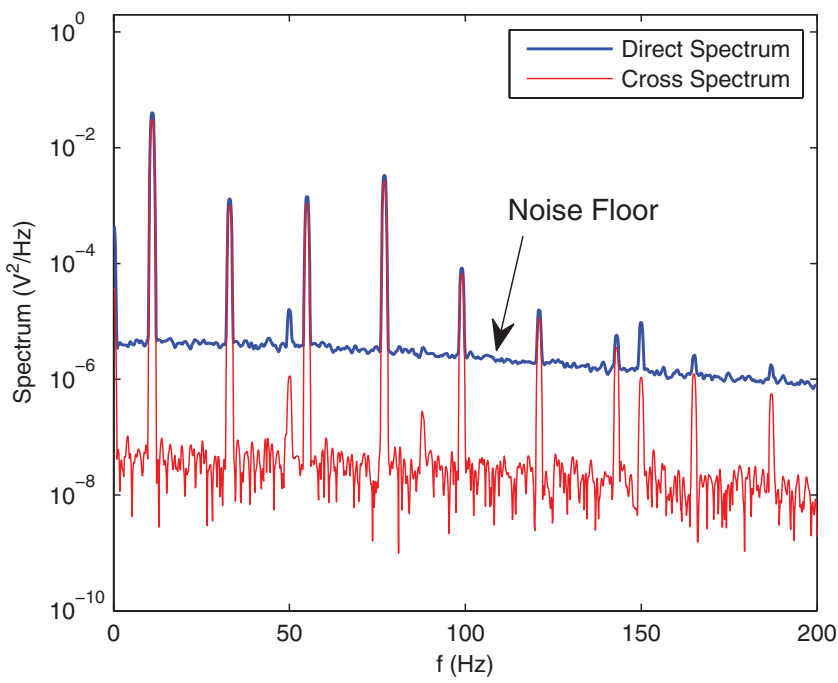

FIG. 4. A comparison between the direct power spectrum estimate and the cross power spectrum estimate. Both measurements used 100 averages $(\mathrm{M}=100)$. 

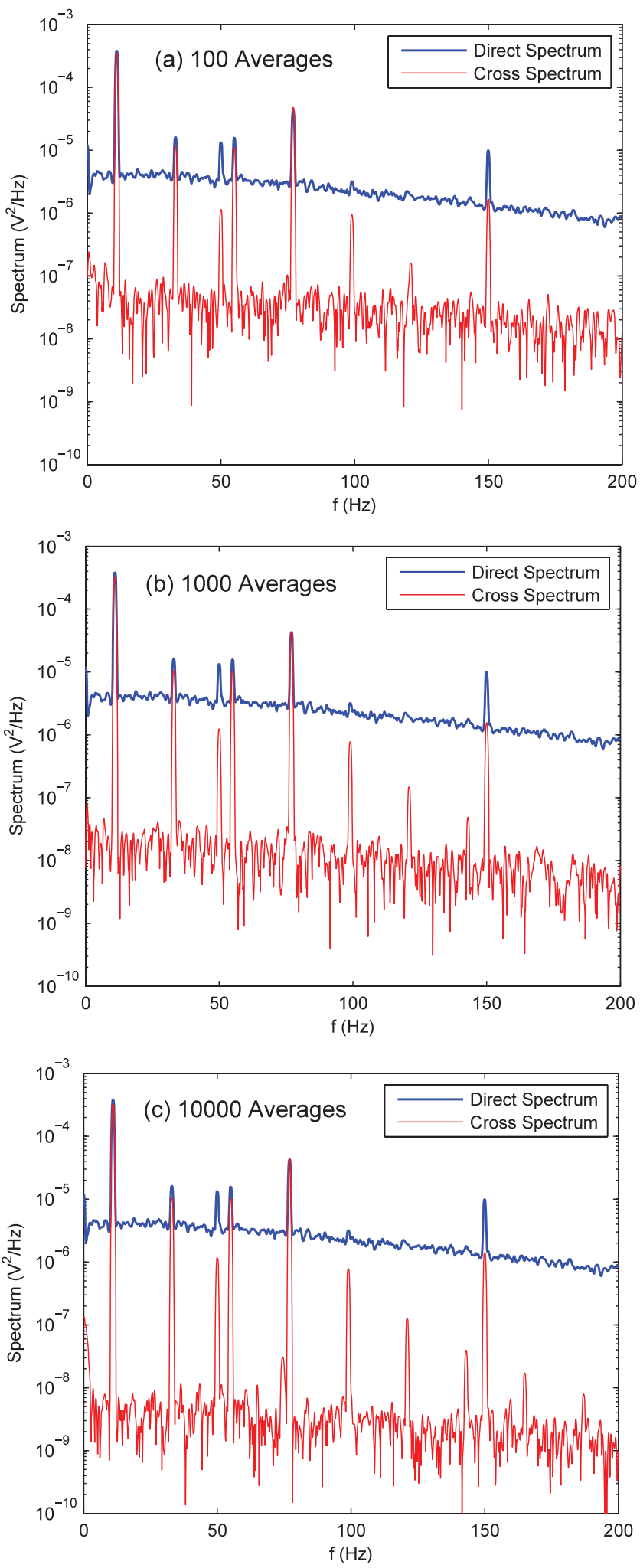

FIG. 5. A comparison between the direct power spectrum and cross power spectrum for (a)100, (b)1000, and (c) 10000 averages.

and all of the harmonics are identifiable. As predicted in Sec. VI B, the estimate variance is observed to be inversely proportional to $\sqrt{M}$. That is, by increasing the number of averages by a factor of 100 , the effective noise floor is reduced by a factor of 10 .
With 10000 averages, the effective noise floor of the cross power spectrum is approximately $3.8 \times 10^{-9} \mathrm{~V}^{2} / \mathrm{Hz}$, or three orders of magnitude better than the direct power spectrum estimate. The equivalent position noise floor is $270 \mathrm{~nm} / \sqrt{\mathrm{Hz}}$ which is an exceptional result considering that the only difference to a standard measurement is the addition of an auxiliary strain gauge.

\section{CONCLUSIONS}

This article reports a simple technique for recovering the power spectrum of a signal from two noisy measurements. If the noise from each measurement is uncorrelated, the power spectral density of the underlying signal can be estimated by computing the cross power spectral density of the two measurements.

The Welch technique was considered as an estimator for the cross power spectral density. This approach is shown to provide a biased but consistent estimate of the underlying spectrum. In addition, the variance of the estimate was shown to be inversely proportional to the square-root of data sets available. The effective noise floor can be reduced arbitrarily by increasing the number of recorded data sets (averages).

The proposed technique was applied experimentally to estimate the vibration spectrum of a piezoelectric cantilever. By using two sensors and 10000 data sets, the effective resolution of the power spectrum was increased by three orders of magnitude. In effect, the vibration spectrum obtained from two low-cost strain sensors was comparable in resolution to a state-of-the-art capacitive or optical position sensor.

The dual sensor technique could potentially be applied to a wide range of scientific and industrial signal recovery problems where an additional sensor is feasible. A reduction of the estimate variance by three orders of magnitude could potentially reveal new discoveries in astrophysical measurements, chemical analysis, and geological surveying.

\section{ACKNOWLEDGMENTS}

This research was supported by the Australian Research Council.

${ }^{1}$ S. M. Kay, Modern Spectral Estimation, Theory and Application (Prentice Hall, Inc., 1988).

${ }^{2}$ S. L. Marple, Jr., Digital Spectral Analysis with Applications (Prentice Hall, Inc., 1987).

${ }^{3}$ T.-P. Jung, S. Makeig, M. Stensmo, and T. Sejnowski, IEEE Trans. Biomed. Eng. 44, 60 (1997).

${ }^{4}$ J. Li and P. Stoica, IEEE Trans. Signal Process. 44, 1469 (1996).

${ }^{5}$ A. Takahata, E. Nadalin, R. Ferrari, L. Duarte, R. Suyama, R. Lopes, J. Romano, and M. Tygel, IEEE Signal Process. Mag. 29, 27 (2012).

${ }^{6}$ G. Li, B. Miao, Q. Hu, and G. Qin, Phys. Rev. Lett. 106, 125001 (2011).

${ }^{7}$ Ocean Wave Energy. Current Status and Future Perspectives, edited by J. Cruz (Springer-Verlag, 2008).

${ }^{8}$ A. J. Fleming, Rev. Sci. Instrum. 83, 086101 (2012).

${ }^{9}$ A. J. Fleming, S. S. Aphale, and S. O. R. Moheimani, IEEE Trans. Nanotechnol. 9, 438 (2010).

${ }^{10}$ B. M. Lansdorp and O. A. Saleh, Rev. Sci. Instrum. 83, 025115 (2012).

${ }^{11}$ S. Kay and S. L. Marple Jr., Proc. IEEE 69, 1380 (1981).

${ }^{12}$ D. Thomson, Proc. IEEE 70, 1055 (1982).

${ }^{13} \mathrm{P}$. Stoica and K. Moses, Introduction to Spectral Analysis (Prentice Hall, Inc., 1997). 
${ }^{14}$ Y. K. Yong, A. J. Fleming, and S. O. R. Moheimani, IEEE/ASME Trans. Mechatron. 18, 1113 (2013).

${ }^{15}$ A. J. Fleming, IEEE/ASME Trans. Mechatron. 15, 433 (2010).

${ }^{16}$ A. Sebastian and A. Pantazi, IEEE Trans. Control Syst. Technol. 20, 382 (2012).

${ }^{17}$ A. Pantazi, A. Sebastian, G. Cherubini, M. Lantz, H. Pozidis, H. Rothuizen, and E. Eleftheriou, IEEE Trans. Control Syst. Technol. 15, 824 (2007).

${ }^{18}$ L. E. Bengtsson, Rev. Sci. Instrum. 83, 075103 (2012).

${ }^{19}$ M. O. Sonnaillon and F. J. Bonetto, Rev. Sci. Instrum. 76, 024703 (2005).
${ }^{20}$ L. Ljung, System Identification: Theory for the User (Prentice Hall, Upper Saddle River, NJ, 1999)

${ }^{21}$ P. Welch, IEEE Trans. Audio Electroacoust. 15, 70 (1967).

${ }^{22}$ J. G. Proakis and D. G. Manolakis, Digital Signal Processing. Principles, Algorithms, and Applications, 3rd ed. (Prentice Hall, Inc., Upper Saddle River, NJ, 1996).

${ }^{23}$ R. Edwards, Fourier Series: A Modern Introduction (Springer-Verlag, 1979).

${ }^{24}$ A. J. Fleming, Sens. Actuators, A 190, 106 (2013). 